Probl. Anal. Issues Anal. Vol. 6 (24), No. 2, 2017, pp. 3-24

DOI: $10.15393 /$ j3.art.2017.4070

UDC 517.521.2

G. G. Akniyev

\title{
DISCRETE LEAST SQUARES APPROXIMATION OF PIECEWISE-LINEAR FUNCTIONS BY TRIGONOMETRIC POLYNOMIALS
}

\begin{abstract}
Let $N$ be a natural number greater than 1 . Select $N$ uniformly distributed points $t_{k}=2 \pi k / N(0 \leq k \leq$ $\leq N-1)$ on $[0,2 \pi]$. Denote by $L_{n, N}(f)=L_{n, N}(f, x)(1 \leq$ $\leq n \leq N / 2$ ) the trigonometric polynomial of order $n$ possessing the least quadratic deviation from $f$ with respect to the system $\left\{t_{k}\right\}_{k=0}^{N-1}$. In this article approximation of functions by the polynomials $L_{n, N}(f, x)$ is considered. Special attention is paid to approximation of $2 \pi$-periodic functions $f_{1}$ and $f_{2}$ by the polynomials $L_{n, N}(f, x)$, where $f_{1}(x)=|x|$ and $f_{2}(x)=\operatorname{sign} x$ for $x \in$ $\in[-\pi, \pi]$. For the first function $f_{1}$ we show that instead of the estimation $\left|f_{1}(x)-L_{n, N}\left(f_{1}, x\right)\right| \leq c \ln n / n$ which follows from the well-known Lebesgue inequality for the polynomials $L_{n, N}(f, x)$ we found an exact order estimation $\left|f_{1}(x)-L_{n, N}\left(f_{1}, x\right)\right| \leq c / n$ $(x \in \mathbb{R})$ which is uniform with respect to $1 \leq n \leq N / 2$. Moreover, we found a local estimation $\left|f_{1}(x)-L_{n, N}\left(f_{1}, x\right)\right| \leq c(\varepsilon) / n^{2}$ $(|x-\pi k| \geq \varepsilon)$ which is also uniform with respect to $1 \leq n \leq$ $\leq N / 2$. For the second function $f_{2}$ we found only a local estimation $\left|f_{2}(x)-L_{n, N}\left(f_{2}, x\right)\right| \leq c(\varepsilon) / n(|x-\pi k| \geq \varepsilon)$ which is uniform with respect to $1 \leq n \leq N / 2$. The proofs of these estimations are based on comparing of approximating properties of discrete and continuous finite Fourier series.
\end{abstract}

Key words: function approximation, trigonometric polynomials, Fourier series

2010 Mathematical Subject Classification: 41A25

1. Introduction. Let $N$ be a natural number greater then 1 . Let $\left\{t_{k}\right\}_{k=0}^{N-1}$ be a set of points on $[0,2 \pi]$ where $t_{k}=2 \pi k / N$. Denote by $L_{n, N}(f)=L_{n, N}(f, x)(1 \leq n \leq\lfloor N / 2\rfloor)$ a trigonometric polynomial of 
order $n$ possessing the least quadratic deviation from the function $f$ with respect to the system $\left\{t_{k}\right\}_{k=0}^{N-1}$. In other words, the minimum of the sums $\sum_{k=0}^{N-1}\left|f\left(t_{k}\right)-T_{n}\left(t_{k}\right)\right|^{2}$ on the set of trigonometric polynomials $T_{n}$ of order $n$ is attained when $T_{n}=L_{n, N}(f)$. In particular, $L_{\lfloor N / 2\rfloor, N}\left(f, t_{k}\right)=$ $=f\left(t_{k}\right)$. It is easy to show (see [12]) that for $n<N / 2$ the polynomial $L_{n, N}(f, x)$ can be represented as

$$
L_{n, N}(f, x)=\sum_{\nu=-n}^{n} c_{\nu}^{(N)}(f) e^{i \nu x}, \quad c_{\nu}^{(N)}(f)=\frac{1}{N} \sum_{k=0}^{N-1} f\left(t_{k}\right) e^{-i \nu t_{k}},
$$

and for $n=N / 2($ for an even $\mathrm{N})$

$$
L_{N / 2, N}(f, x)=L_{N / 2-1, N}(f, x)+a_{N / 2}^{(N)}(f) \cos N / 2(x-u),
$$

where

$$
a_{n}^{(2 n)}(f)=a_{N / 2}^{(N)}(f)=\frac{1}{N} \sum_{k=0}^{N-1} f\left(t_{k}\right) \cos N / 2\left(t_{k}-u\right) .
$$

To read more about function approximation by trigonometric polynomials see [1, 3, 5, 6, 8 - 11], [13. In this article we obtain estimations for $\left|L_{n, N}\left(f_{1}, x\right)-f_{1}(x)\right|$ and $\left|L_{n, N}\left(f_{2}, x\right)-f_{2}(x)\right|$ as $n, N \rightarrow \infty$, where $f_{1}(x)=$ $=|x|, f_{2}(x)=\operatorname{sign} x, x \in[-\pi, \pi]$. The following theorems are proved:

Theorem 1. Let $f_{1}(x)=|x|, x \in[-\pi, \pi]$ and $n \leq\lfloor N / 2\rfloor$. The following estimations hold:

$$
\begin{gathered}
\left|L_{n, N}\left(f_{1}, x\right)-f_{1}(x)\right| \leq c / n, \quad x \in[-\pi, \pi], \\
\left|L_{n, N}\left(f_{1}, x\right)-f_{1}(x)\right| \leq c(\varepsilon) / n^{2}, \quad x \in \Delta^{I}(\varepsilon) .
\end{gathered}
$$

Theorem 2. Let $f_{2}(x)=\operatorname{sign} x, x \in[-\pi, \pi]$ and $n \leq\lfloor N / 2\rfloor$. The following estimation holds:

$$
\left|L_{n, N}(f, x)-f(x)\right| \leq c(\varepsilon) / n, \quad x \in \Delta^{I}(\varepsilon) .
$$

We begin with some notation. Denote by

$$
c_{k}(f)=\frac{1}{2 \pi} \int_{-\pi}^{\pi} f(t) e^{-i \nu t} d t, \quad k \in \mathbb{Z},
$$


the Fourier coefficients of a function $f$, and by

$$
f(x)=\sum_{k \in \mathbb{Z}} c_{k}(f) e^{i k x}, \quad S_{n}(f, x)=\sum_{k=-n}^{n} c_{k}(f) e^{i k x}
$$

the Fourier series of a function $f$ and its partial sum of order $n$, respectively. Denote by $\Delta^{I}(\varepsilon)$ the set $[-\pi+\varepsilon,-\varepsilon] \cup[\varepsilon, \pi-\varepsilon]$, where $0<\varepsilon<\pi / 2$. By $c$ and $c(\varepsilon)$ we denote some positive constants that depend only on specified parameters; these constants may be different in different places.

Lemma 1. 12] If the Fourier series of $f$ converges at the points $t_{k}=$ $=u+2 k \pi / N$, then the representation

$$
L_{n, N}(f, x)=S_{n}(f, x)+R_{n, N}(f, x),
$$

where

$$
R_{n, N}(f, x)=\frac{2}{\pi} \sum_{\mu=1}^{\infty} \int_{-\pi}^{\pi} D_{n}(x-t) \cos \mu N(u-t) f(t) d t
$$

holds true when $2 n<N$.

The following estimation follows from this lemma:

$$
\left|L_{n, N}(f, x)-f(x)\right| \leq\left|S_{n}(f, x)-f(x)\right|+\left|R_{n, N}(f, x)\right|, \quad n<N / 2 .
$$

Let us consider the case $2 n=N$. From (1) and (4)

$$
\begin{gathered}
\left|L_{n, 2 n}(f, x)-f(x)\right| \leq \\
\leq\left|S_{n-1}(f, x)-f(x)\right|+\left|R_{n-1,2 n}(f, x)\right|+\left|a_{n}^{(2 n)}(f)\right|, \quad n=N / 2 .
\end{gathered}
$$

From (4) and (5) we can see that estimation of the values $\mid S_{n}\left(f_{1}, x\right)-$ $-f_{1}(x)|,| S_{n}\left(f_{2}, x\right)-f_{2}(x)|,| R_{n, N}\left(f_{1}, x\right)|,| R_{n, N}\left(f_{2}, x\right)|,| a_{n}^{(2 n)}\left(f_{1}\right) \mid$, and $\left|a_{n}^{(2 n)}\left(f_{2}\right)\right|$ implies an estimation $\left|L_{n, N}(f, x)-f(x)\right|$ for the functions $f_{1}$ and $f_{2}$.

2. Estimations for $\left|S_{n}\left(f_{1}, x\right)-f_{1}(x)\right|$ and $\left|S_{n}\left(f_{2}, x\right)-f_{2}(x)\right|$.

Lemma 2. For the value $\left|S_{n}\left(f_{1}, x\right)-f_{1}(x)\right|$, where $f_{1}(x)=|x|, x \in$ $\in[-\pi, \pi]$, we have the following estimations:

$$
\left|S_{n}\left(f_{1}, x\right)-f_{1}(x)\right| \leq c / n, \quad x \in[-\pi, \pi],
$$




$$
\left|S_{n}\left(f_{1}, x\right)-f_{1}(x)\right| \leq c(\varepsilon) / n^{2}, \quad x \in \Delta^{I}(\varepsilon) .
$$

Proof. Using [2, p.443] or [4, p.690], we can get the following representation for $f_{1}(x)$ on $[-\pi, \pi]$ :

$$
f_{1}(x)=|x|=\frac{\pi}{2}-\frac{4}{\pi} \sum_{k=1}^{\infty} \frac{\cos (2 k-1) x}{(2 k-1)^{2}}, \quad x \in[-\pi, \pi] .
$$

From the above we can obtain estimation (6) (using the fact that $|\cos (2 k-1) x| \leq 1)$. Also, if we apply the Abel transformation to the above equation, we get (7).

Lemma 3. For the value $\left|S_{n}\left(f_{2}, x\right)-f_{2}(x)\right|$, where $f_{2}(x)=\operatorname{sign} x, x \in$ $\in[-\pi, \pi]$ we have the following estimation:

$$
\left|S_{n}\left(f_{2}, x\right)-f_{2}(x)\right| \leq c(\varepsilon) / n, \quad x \in \Delta^{I}(\varepsilon) .
$$

The proof of this lemma is obtained in [7].

3. An estimation for $R_{n, N}\left(f_{1}, x\right)$. The following lemma takes place.

Lemma 4. For $R_{n, N}\left(f_{1}, x\right), n<N / 2$ the following estimations hold:

$$
\begin{gathered}
\left|R_{n, N}\left(f_{1}, x\right)\right| \leq \frac{\pi}{n}\left(4+\frac{1}{2 n}\right) \leq \frac{c}{n}, \quad x \in[-\pi, \pi], \\
\left|R_{n, N}\left(f_{1}, x\right)\right| \leq \frac{\pi}{n^{2}}\left(\frac{1}{6}+\frac{4}{|\sin \varepsilon|}\right) \leq \frac{c(\varepsilon)}{n^{2}}, \quad x \in \Delta^{I}(\varepsilon) .
\end{gathered}
$$

Note that the estimation for remainder (3) for the function $f_{1}$ has the following form:

$$
R_{n, N}\left(f_{1}, x\right)=\frac{2}{\pi} \sum_{\mu=1}^{\infty} \int_{-\pi}^{\pi}|t| D_{n}(x-t) \cos \mu N(u-t) d t
$$

where

$$
D_{n}(x-t)=1 / 2+\sum_{k=1}^{n} \cos k(x-t) .
$$

From above we have

$$
\left|R_{n, N}\left(f_{1}, x\right)\right| \leq\left|R_{n, N}^{1}\left(f_{1}, x\right)\right|+\left|R_{n, N}^{2}\left(f_{1}, x\right)\right|,
$$


where

$$
\begin{gathered}
R_{n, N}^{1}\left(f_{1}, x\right)=\frac{1}{\pi} \sum_{\mu=1}^{\infty} \int_{-\pi}^{\pi}|t| \cos \mu N(u-t) d t \\
R_{n, N}^{2}\left(f_{1}, x\right)=\frac{2}{\pi} \sum_{\mu=1}^{\infty} \int_{-\pi}^{\pi}|t| \sum_{k=1}^{n} \cos k(x-t) \cos \mu N(u-t) d t .
\end{gathered}
$$

Lemma 5. The value $\left|R_{n, N}^{1}\left(f_{1}, x\right)\right|$ has the following estimation:

$$
\left|R_{n, N}^{1}\left(f_{1}, x\right)\right| \leq c / n^{2}, \quad x \in[-\pi, \pi] .
$$

Proof. Using the formula

$$
\cos (\mu N u-\mu N t)=\cos \mu N u \cos \mu N t+\sin \mu N u \sin \mu N t
$$

we can rewrite $(9$ ) as follows:

$$
R_{n, N}^{1}\left(f_{1}, x\right)=\frac{2}{\pi} \sum_{\mu=1}^{\infty} \cos \mu N u \int_{0}^{\pi} t \cos \mu N t d t .
$$

Then we calculate the integral using the previous equation:

$$
\int_{0}^{\pi} t \cos \mu N t d t=\frac{(-1)^{\mu N}-1}{(\mu N)^{2}} .
$$

From these equations we get

$$
R_{n, N}^{1}\left(f_{1}, x\right)=\frac{2}{\pi N^{2}} \sum_{\mu=1}^{\infty} \frac{\cos \mu N u}{\mu^{2}}\left((-1)^{\mu N}-1\right)
$$

or

$$
R_{n, N}^{1}\left(f_{1}, x\right)= \begin{cases}0, & N=2 l \\ -\frac{4}{\pi N^{2}} \sum_{\mu=1}^{\infty} \frac{\cos (2 \mu-1) N u}{(2 \mu-1)^{2}}, & N=2 l+1 .\end{cases}
$$

Now we can estimate $\left|R_{n, N}^{1}\left(f_{1}, x\right)\right|$ :

$$
\left|R_{n, N}^{1}\left(f_{1}, x\right)\right| \leq \frac{4}{\pi N^{2}} \sum_{\mu=1}^{\infty} \frac{1}{(2 \mu-1)^{2}}
$$


It is known that

$$
\sum_{\mu=1}^{\infty} \frac{1}{(2 \mu-1)^{2}}=\frac{\pi^{2}}{8}
$$

So, using 12 we obtain

$$
\left|R_{n, N}^{1}\left(f_{1}, x\right)\right| \leq \frac{\pi}{2 N^{2}} \leq \frac{\pi}{8 n^{2}}
$$

Lemma 6. The value $\left|R_{n, N}^{2}\left(f_{1}, x\right)\right|$ has the following estimation for $x \in$ $\in[-\pi, \pi]:$

$$
\left|R_{n, N}^{2}\left(f_{1}, x\right)\right| \leq \frac{c}{n}, \quad x \in[-\pi, \pi] .
$$

Before proving Lemma 6, we prove another one:

Lemma 7. The following estimations take place:

$$
\begin{gathered}
\left|\sum_{i=0}^{k} \cos (2 i-1) x\right| \leq \frac{1}{2|\sin x|}, \quad\left|\sum_{i=0}^{k} \cos 2 i x\right| \leq \frac{1}{|\sin x|} \\
\left|\sum_{i=1}^{k} \sin (2 i+1) x\right| \leq \frac{1}{|\sin x|}, \quad\left|\sum_{i=0}^{k-1} \sin 2 i x\right| \leq \frac{1}{|\sin x|}
\end{gathered}
$$

Proof. After some simple transforms we obtain the following:

$$
\begin{gathered}
\sum_{i=1}^{k} \cos (2 i-1) x=\frac{1}{\sin x} \sum_{i=1}^{k} \sin x \cos (2 i-1) x= \\
=\frac{1}{2 \sin x} \sum_{i=1}^{k}(\sin (x-(2 i-1) x)+\sin (x+(2 i-1) x)= \\
=\frac{1}{2 \sin x} \sum_{i=1}^{k}(\sin 2 i x-\sin 2(i-1) x)=\frac{\sin 2 k x}{2 \sin x} .
\end{gathered}
$$

Likewise, obtain

$$
\sum_{i=1}^{k} \cos 2 i x=\frac{1}{\sin x} \sum_{i=1}^{k} \sin x \cos 2 i x=\frac{\sin (2 k+1) x-\sin x}{2 \sin x},
$$




$$
\begin{gathered}
\sum_{i=1}^{k} \sin (2 i+1) x=\frac{\cos 2 x-\cos (2 k+2) x}{2 \sin x}, \\
\sum_{i=1}^{k} \sin 2 i x=\frac{\cos x-\cos (2 k+1) x}{2 \sin x} .
\end{gathered}
$$

Now the proof is complete.

Proof. (Lemma 6) From (11) and

$$
\cos k(x-t)=\cos k x \cos k t+\sin k x \sin k t
$$

(10) can be rewritten as follows:

$$
\begin{aligned}
R_{n, N}^{2}\left(f_{1}, x\right)=\frac{4}{\pi} \sum_{\mu=1}^{\infty} \cos \mu N u \sum_{k=1}^{n} \cos k x \int_{0}^{\pi} t \cos k t \cos \mu N t d t+ \\
\quad+\frac{4}{\pi} \sum_{\mu=1}^{\infty} \sin \mu N u \sum_{k=1}^{n} \sin k x \int_{0}^{\pi} t \sin k t \sin \mu N t d t
\end{aligned}
$$

From above and the formulas

$$
\begin{gathered}
\cos k t \cos \mu N t=1 / 2(\cos (\mu N-k) t+\cos (\mu N+k) t), \\
\sin k t \sin \mu N t=1 / 2(\cos (\mu N-k) t-\cos (\mu N+k) t)
\end{gathered}
$$

we can obtain

$$
\begin{aligned}
& R_{n, N}^{2}\left(f_{1}, x\right)=\frac{2}{\pi} \sum_{\mu=1}^{\infty} \cos \mu N u \times \\
& \times \sum_{k=1}^{n} \cos k x\left(\int_{0}^{\pi} t \cos (\mu N-k) t d t+\int_{0}^{\pi} t \cos (\mu N+k) t d t\right)+ \\
& \quad+\frac{2}{\pi} \sum_{\mu=1}^{\infty} \sin \mu N u \times \\
& \quad \times \sum_{k=1}^{n} \sin k x\left(\int_{0}^{\pi} t \cos (\mu N-k) t d t-\int_{0}^{\pi} t \cos (\mu N+k) t d t\right) .
\end{aligned}
$$


After calculating the integrals, we have

$$
\begin{aligned}
& R_{n, N}^{2}\left(f_{1}, x\right)=\frac{2}{\pi} \sum_{\mu=1}^{\infty} \cos \mu N u \times \\
& \times \sum_{k=1}^{n}\left((-1)^{\mu N+k}-1\right) \cos k x\left(\frac{1}{(\mu N-k)^{2}}+\frac{1}{(\mu N+k)^{2}}\right)+ \\
& \quad+\frac{2}{\pi} \sum_{\mu=1}^{\infty} \sin \mu N u \times \\
& \quad \times \sum_{k=1}^{n}\left((-1)^{\mu N+k}-1\right) \sin k x\left(\frac{1}{(\mu N-k)^{2}}-\frac{1}{(\mu N+k)^{2}}\right) .
\end{aligned}
$$

We can rewrite this as follows:

$$
\begin{aligned}
&\left|R_{n, N}\left(f_{1}, x\right)\right| \leq \frac{4}{\pi} \sum_{\mu=1}^{\infty} \sum_{k=1}^{n}\left(\frac{1}{(\mu N-k)^{2}}+\frac{1}{(\mu N+k)^{2}}\right)+ \\
&+\frac{4}{\pi} \sum_{\mu=1}^{\infty} \sum_{k=1}^{n}\left(\frac{1}{(\mu N-k)^{2}}-\frac{1}{(\mu N+k)^{2}}\right) \leq \\
& \leq \frac{12}{\pi} \sum_{\mu=1}^{\infty} \sum_{k=1}^{n} \frac{1}{(\mu N-k)^{2}} \leq \frac{12}{\pi N} \sum_{\mu=1}^{\infty} \frac{1}{\mu^{2}} \sum_{k=1}^{n} \frac{1}{N\left(1-\frac{n}{\mu N}\right)^{2}} \leq \frac{4 \pi}{N} \leq \frac{2 \pi}{n} .
\end{aligned}
$$

Lemma 8. The value $\left|R_{n, N}^{2}\left(f_{1}, x\right)\right|$ has the following estimation for $x \in$ $\in \Delta^{I}(\varepsilon):$

$$
\left|R_{n, N}^{2}\left(f_{1}, x\right)\right| \leq \frac{c}{n^{2} \sin \varepsilon}=\frac{c(\varepsilon)}{n^{2}}, \quad x \in \Delta^{I}(\varepsilon) .
$$

Proof. To prove this lemma, we use 14). It is easy to show that for even $N$

$$
R_{n, N}^{2}\left(f_{1}, x\right)=-\frac{4}{\pi} \sum_{\mu=1}^{\infty} \cos \mu N u A_{n}^{\mu, N}(x)-\frac{4}{\pi} \sum_{\mu=1}^{\infty} \sin \mu N u B_{n}^{\mu, N}(x),
$$

where

$$
A_{n}^{\mu, N}(x)=\sum_{k=1}^{\lceil n / 2\rceil} \cos (2 k-1) x\left(\frac{1}{(\mu N-(2 k-1))^{2}}+\frac{1}{(\mu N+(2 k-1))^{2}}\right),
$$




$$
B_{n}^{\mu, N}(x)=\sum_{k=1}^{\lceil n / 2\rceil} \sin (2 k-1) x\left(\frac{1}{(\mu N-(2 k-1))^{2}}-\frac{1}{(\mu N+(2 k-1))^{2}}\right) .
$$

And, therefore

$$
\left|R_{n, N}^{2}\left(f_{1}, x\right)\right|=\frac{4}{\pi} \sum_{\mu=1}^{\infty}\left|A_{n}^{\mu, N}(x)\right|+\frac{4}{\pi} \sum_{\mu=1}^{\infty}\left|B_{n}^{\mu, N}(x)\right| .
$$

Consider the values $\left|A_{n}^{\mu, N}(x)\right|$ and $\left|B_{n}^{\mu, N}(x)\right|$. Apply the Abel transformation for $A_{n}^{\mu, N}(x)$ :

$$
\begin{gathered}
A_{n}^{\mu, N}(x)=\left(\frac{1}{(\mu N-(2\lceil n / 2\rceil-1))^{2}}+\frac{1}{(\mu N+(2\lceil n / 2\rceil-1))^{2}}\right) \times \\
\times \sum_{i=1}^{\lceil n / 2\rceil} \cos (2 i-1) x-\sum_{k=1}^{\lceil n / 2\rceil-1}\left[\left(\frac{1}{(\mu N-(2 k+1))^{2}}+\frac{1}{(\mu N+(2 k+1))^{2}}\right)-\right. \\
\left.-\left(\frac{1}{(\mu N-(2 k-1))^{2}}+\frac{1}{(\mu N+(2 k-1))^{2}}\right)\right] \sum_{i=1}^{k} \cos (2 i-1) x .
\end{gathered}
$$

From this we get the following estimation:

$$
\begin{gathered}
\left|A_{n}^{\mu, N}(x)\right| \leq\left(\frac{1}{(\mu N-(2\lceil n / 2\rceil-1))^{2}}+\frac{1}{(\mu N+(2\lceil n / 2\rceil-1))^{2}}\right) \times \\
\times\left|\sum_{i=1}^{\lceil n / 2\rceil} \cos (2 i-1) x\right|+\sum_{k=1}^{\lceil n / 2\rceil-1}\left[\left(\frac{1}{(\mu N-(2 k+1))^{2}}+\frac{1}{(\mu N+(2 k+1))^{2}}\right)-\right. \\
\left.-\left(\frac{1}{(\mu N-(2 k-1))^{2}}+\frac{1}{(\mu N+(2 k-1))^{2}}\right)\right]\left|\sum_{i=0}^{k} \cos (2 i-1) x\right| .
\end{gathered}
$$

Using Lemma 7, we can rewrite the obtained estimation:

$$
\begin{aligned}
\left|A_{n}^{\mu, N}(x)\right| \leq \frac{2}{|\sin x|} & \left(\frac{1}{(\mu N-(2\lceil n / 2\rceil-1))^{2}}+\right. \\
& \left.+\frac{1}{(\mu N+(2\lceil n / 2\rceil-1))^{2}}\right) \leq \frac{10}{(\mu N)^{2}|\sin x|}
\end{aligned}
$$


Using the similar approach, we get an estimation for $\left|B_{n}^{\mu, N}(x)\right|$ :

$$
\left|B_{n}^{\mu, N}(x)\right| \leq \frac{8}{3 \mu^{3} N^{2}|\sin x|} .
$$

Using 15, (16), and (17) we can write

$$
\begin{gathered}
\left|R_{n, N}^{2}\left(f_{1}, x\right)\right| \leq \frac{4}{\pi} \sum_{\mu=1}^{\infty}\left|A_{n}^{\mu, N}(x)\right|+\frac{4}{\pi} \sum_{\mu=1}^{\infty}\left|B_{n}^{\mu, N}(x)\right| \leq \\
\leq \frac{4}{\pi} \sum_{\mu=1}^{\infty} \frac{10}{(\mu N)^{2}|\sin x|}+\frac{4}{\pi} \sum_{\mu=1}^{\infty} \frac{8}{3 \mu^{3} N^{2}|\sin x|} \leq \frac{26}{N^{2}|\sin x|}, \quad N=2 l .
\end{gathered}
$$

If $N$ is odd, then we can rewrite 14 as follows:

$$
\begin{aligned}
& R_{n, N}^{2}\left(f_{1}, x\right)= \\
& =-\frac{4}{\pi} \sum_{\mu=1}^{\infty} C_{n}^{\mu, N}(x) \cos (2 \mu-1) N u-\frac{4}{\pi} \sum_{\mu=1}^{\infty} D_{n}^{\mu, N}(x) \sin (2 \mu-1) N u- \\
& \quad-\frac{4}{\pi} \sum_{\mu=1}^{\infty} E_{n}^{\mu, N}(x) \cos 2 \mu N u-\frac{4}{\pi} \sum_{\mu=1}^{\infty} F_{n}^{\mu, N}(x) \sin 2 \mu N u,
\end{aligned}
$$

where

$$
\begin{gathered}
C_{n}^{\mu, N}(x)=\sum_{k=1}^{\lfloor n / 2\rfloor} \cos 2 k x\left(\frac{1}{((2 \mu-1) N-2 k)^{2}}+\frac{1}{((2 \mu-1) N+2 k)^{2}}\right), \\
D_{n}^{\mu, N}(x)=\sum_{k=1}^{\lfloor n / 2\rfloor} \sin 2 k x\left(\frac{1}{((2 \mu-1) N-2 k)^{2}}-\frac{1}{((2 \mu-1) N+2 k)^{2}}\right), \\
E_{n}^{\mu, N}(x)=\sum_{k=1}^{\lceil n / 2\rceil} \cos (2 k-1) x\left(\frac{1}{(2 \mu N-(2 k-1))^{2}}+\frac{1}{(2 \mu N+(2 k-1))^{2}}\right), \\
F_{n}^{\mu, N}(x)=\sum_{k=1}^{\lceil n / 2\rceil} \sin (2 k-1) x\left(\frac{1}{(2 \mu N-(2 k-1))^{2}}-\frac{1}{(2 \mu N+(2 k-1))^{2}}\right) .
\end{gathered}
$$




$$
\text { And } \begin{aligned}
\left|R_{n, N}^{2}\left(f_{1}, x\right)\right| \leq \frac{4}{\pi} \sum_{\mu=1}^{\infty} \mid & C_{n}^{\mu, N}(x)\left|+\frac{4}{\pi} \sum_{\mu=1}^{\infty}\right| D_{n}^{\mu, N}(x) \mid+ \\
& +\frac{4}{\pi} \sum_{\mu=1}^{\infty}\left|E_{n}^{\mu, N}(x)\right|+\frac{4}{\pi} \sum_{\mu=1}^{\infty}\left|F_{n}^{\mu, N}(x)\right| .
\end{aligned}
$$

Using the Abel transformation, we can easily obtain these estimations:

$$
\begin{gathered}
\left|C_{n}^{\mu, N}(x)\right| \leq \frac{10}{|\sin x| \mu^{2} N^{2}}, \quad\left|D_{n}^{\mu, N}(x)\right| \leq \frac{8}{|\sin x| \mu^{2} N^{2}} \\
\left|E_{n}^{\mu, N}(x)\right| \leq \frac{2}{|\sin x| \mu^{2} N^{2}}, \quad\left|F_{n}^{\mu, N}(x)\right| \leq \frac{8}{9|\sin x| \mu^{2} N^{2}} .
\end{gathered}
$$

From this we have

$$
\left|R_{n, N}^{2}\left(f_{1}, x\right)\right| \leq \frac{14 \pi}{N^{2}|\sin x|}, \quad N=2 l+1 .
$$

From 18 and 19 we have (for every $N \geq 2$ )

$$
\left|R_{n, N}^{2}\left(f_{1}, x\right)\right| \leq \frac{14 \pi}{N^{2}|\sin x|} \leq \frac{7 \pi}{2 n^{2}|\sin x|} \leq \frac{7 \pi}{2 n^{2}|\sin \varepsilon|}, \quad x \in \Delta^{I}(\varepsilon) .
$$

4. An estimation for $a_{n}^{(2 n)}\left(f_{1}\right)$. When $2 n=N$ from $(2)$ we have the following:

$$
a_{n}^{(2 n)}(f)=\frac{1}{2 n} \sum_{k=0}^{2 n-1} f\left(t_{k}\right) \cos \pi k=\frac{1}{2 n} \sum_{k=0}^{2 n-1}(-1)^{k} f\left(u+\frac{\pi k}{n}\right) .
$$

Subtract $\frac{\pi}{n}$ from $u$ to get (provided that $f$ is $2 \pi$ periodic function)

$$
\begin{gathered}
\frac{1}{2 n} \sum_{k=0}^{2 n-1}(-1)^{k} f\left(\left(u-\frac{\pi}{n}\right)+\frac{\pi k}{n}\right)=\frac{1}{2 n} \sum_{k=0}^{2 n-1}(-1)^{k} f\left(u+\frac{\pi(k-1)}{n}\right)= \\
=\frac{1}{2 n} \sum_{k=1}^{2 n}(-1)^{k-1} f\left(u+\frac{\pi k}{n}\right)=-\frac{1}{2 n} \sum_{k=1}^{2 n}(-1)^{k} f\left(u+\frac{\pi k}{n}\right)= \\
=-\frac{1}{2 n} \sum_{k=1}^{2 n-1}(-1)^{k} f\left(u+\frac{\pi k}{n}\right)+(-1)^{2 n} f(u+2 \pi)= \\
=-\frac{1}{2 n} \sum_{k=0}^{2 n-1}(-1)^{k} f\left(u+\frac{\pi k}{n}\right)=-a_{n}^{(2 n)}(f) .
\end{gathered}
$$


Therefore, if we subtract or add $\frac{\pi l}{n}$ from $u$, we get

$$
a_{n}^{(2 n)}(f)=\frac{(-1)^{l}}{2 n} \sum_{k=0}^{2 n-1}(-1)^{k} f\left(\left(u+\frac{\pi l}{n}\right)+\frac{\pi k}{n}\right) .
$$

In other words, adding $\frac{\pi l}{n}(l \in \mathbb{Z})$ to $u$ does not change the value of $\left|a_{n}^{(2 n)}(f)\right|$, so we can assume, without loss of generality, that $0 \leq u<\frac{\pi}{n}$. It holds for both $\left|a_{n}^{(2 n)}\left(f_{1}\right)\right|$ and $\left|a_{n}^{(2 n)}\left(f_{2}\right)\right|$.

Lemma 9. The following estimation takes place:

$$
\left|a_{n}^{(2 n)}\left(f_{1}\right)\right| \leq \frac{c}{n^{2}}, \quad x \in[-\pi, \pi] .
$$

Proof. It is easy to show that

$$
\begin{gathered}
a_{n}^{(2 n)}\left(f_{1}\right)=\frac{1}{2 n} \sum_{k=-n}^{n-1}(-1)^{k}\left|\frac{\pi k}{n}+u\right|, \quad 0 \leq u<\frac{\pi}{n} . \\
a_{n}^{(2 n)}\left(f_{1}\right)=\frac{1}{2 n} \sum_{k=-n}^{n-1}(-1)^{k}\left|\frac{\pi k}{n}+u\right|= \\
=\frac{1}{2 n} \sum_{k=-n}^{-1}(-1)^{k}\left(-\frac{\pi k}{n}-u\right)+\frac{1}{2 n} \sum_{k=0}^{n-1}(-1)^{k}\left(\frac{\pi k}{n}+u\right)= \\
=\frac{1}{2 n} \sum_{k=1}^{n}(-1)^{k}\left(\frac{\pi k}{n}-u\right)+\frac{1}{2 n} \sum_{k=0}^{n-1}(-1)^{k}\left(\frac{\pi k}{n}+u\right)= \\
=\frac{1}{2 n} \sum_{k=1}^{n}(-1)^{k}\left(\frac{\pi k}{n}-u\right)-\frac{1}{2 n} \sum_{k=1}^{n}(-1)^{k}\left(\frac{\pi(k-1)}{n}+u\right)= \\
=\frac{1}{2 n} \sum_{k=1}^{n}(-1)^{k}\left(\frac{\pi k}{n}-\frac{\pi(k-1)}{n}-2 u\right)=\frac{1}{2 n} \sum_{k=1}^{n}(-1)^{k}\left(\frac{\pi}{n}-2 u\right) .
\end{gathered}
$$

Get the following estimation:

$$
\left|a_{n}^{(2 n)}\left(f_{1}\right)\right| \leq\left|\frac{1}{2 n} \sum_{k=1}^{n}(-1)^{k}\left(\frac{\pi}{n}-2 u\right)\right| \leq \frac{1}{2 n}\left|\frac{\pi}{n}-2 u\right| \leq \frac{\pi}{2 n^{2}} .
$$


5. An estimation for $R_{n, N}\left(f_{2}, x\right)$. We prove the following lemma. Lemma 10. The following takes place:

$$
\left|R_{n, N}\left(f_{2}, x\right)\right| \leq \frac{c(\varepsilon)}{n}, \quad x \in \Delta^{I}(\varepsilon) .
$$

To prove this lemma we estimate $\left|R_{n, N}\left(f_{1}, x\right)\right|$ as follows:

$$
\left|R_{n, N}\left(f_{2}, x\right)\right| \leq\left|R_{n, N}^{1}\left(f_{2}, x\right)\right|+\left|R_{n, N}^{2}\left(f_{2}, x\right)\right|
$$

where

$$
\begin{gathered}
R_{n, N}^{1}\left(f_{2}, x\right)=\frac{1}{\pi} \sum_{\mu=1}^{\infty} \int_{-\pi}^{\pi} \cos \mu N(u-t) \operatorname{sign} t d t \\
R_{n, N}^{2}\left(f_{2}, x\right)=\frac{2}{\pi} \sum_{\mu=1}^{\infty} \int_{-\pi}^{\pi} \sum_{k=1}^{n} \cos k(x-t) \cos \mu N(u-t) \operatorname{sign} t d t .
\end{gathered}
$$

First we prove the following lemmas:

Lemma 11. The following takes place:

$$
\left|R_{n, N}^{1}\left(f_{2}, x\right)\right| \leq \frac{c}{n} .
$$

Proof. Using (11) for 22 we have

$$
\begin{aligned}
& R_{n, N}^{1}\left(f_{2}, x\right)=\frac{1}{\pi} \sum_{\mu=1}^{\infty} \int_{-\pi}^{\pi} \cos \mu N(u-t) \operatorname{sign} t d t= \\
& =\frac{1}{\pi} \sum_{\mu=1}^{\infty} \int_{-\pi}^{\pi}(\cos \mu N u \cos \mu N t+\sin \mu N u \sin \mu N t) \operatorname{sign} t d t= \\
& =\frac{1}{\pi} \sum_{\mu=1}^{\infty}\left(\cos \mu N u \int_{-\pi}^{\pi} \cos \mu N t \operatorname{sign} t d t+\sin \mu N u \int_{-\pi}^{\pi} \sin \mu N t \operatorname{sign} t d t\right)= \\
& =\frac{1}{\pi} \sum_{\mu=1}^{\infty} \sin \mu N u \int_{-\pi}^{\pi} \sin \mu N t \operatorname{sign} t d t=\frac{2}{\pi} \sum_{\mu=1}^{\infty} \sin \mu N u \int_{0}^{\pi} \sin \mu N t d t
\end{aligned}
$$


From above and

$$
\int_{0}^{\pi} \sin \mu N t d t=-\left.\frac{\cos \mu N t}{\mu N}\right|_{0} ^{\pi}=\frac{1-(-1)^{\mu N}}{\mu N}
$$

we have

$$
R_{n, N}^{1}\left(f_{2}, x\right)=\frac{2}{\pi N} \sum_{\mu=1}^{\infty}\left(1-(-1)^{\mu N}\right) \frac{\sin \mu N u}{\mu} .
$$

If $N$ is even, then $R_{n, N}^{1}\left(f_{2}, x\right) \equiv 0$, otherwise

$$
R_{n, N}^{1}\left(f_{2}, x\right)=-\frac{4}{\pi N} \sum_{\mu=1}^{\infty} \frac{\sin (2 \mu-1) N u}{2 \mu-1},
$$

where

$$
\left|\frac{4}{\pi} \sum_{\mu=1}^{\infty} \frac{\sin (2 \mu-1) x}{2 \mu-1}\right|=\left|f_{2}(x)\right| \leq 1 .
$$

Therefore

$$
\left|R_{n, N}^{1}\left(f_{2}, x\right)\right| \leq \frac{1}{N} \leq \frac{1}{2 n}
$$

Lemma 12. Denote $\gamma_{n}(x)=\sum_{k=1}^{n}\left(1-(-1)^{k+m}\right) \alpha_{k} \cos k x$, where all $\alpha_{k}>0$, the sequence $\left\{\alpha_{k}\right\}_{k=1}^{n}$ is monotone, and $m=0,1$. Then

$$
\left|\gamma_{n}(x)\right| \leq \frac{c}{|\sin x|}
$$

Proof. Assume that $\alpha_{k}>\alpha_{k+1}$. After applying the Abel transformation we get

$$
\begin{aligned}
& \gamma_{n}(x)=\sum_{k=1}^{n}\left(1-(-1)^{k+m}\right) \alpha_{k} \cos k x= \\
= & \alpha_{n} \sum_{i=1}^{n}\left(1-(-1)^{i+m}\right) \cos i x-\sum_{k=1}^{n-1}\left(\alpha_{k+1}-\alpha_{k}\right) \sum_{i=1}^{k}\left(1-(-1)^{i+m}\right) \cos i x .
\end{aligned}
$$


From the above equation and Lemma 7 we have

$$
\begin{aligned}
&\left|\gamma_{n}(x)\right| \leq \alpha_{n}\left|\sum_{i=1}^{n}\left(1-(-1)^{i+m}\right) \cos i x\right|+ \\
&+\sum_{k=1}^{n-1}\left(\alpha_{k+1}-\alpha_{k}\right)\left|\sum_{i=1}^{k}\left(1-(-1)^{i+m}\right) \cos i x\right| \leq \\
& \leq \frac{1}{|\sin x|}\left(\alpha_{n}+\alpha_{n}-\alpha_{0}\right) \leq \frac{2 \alpha_{n}}{|\sin x|}=\frac{c}{|\sin x|}
\end{aligned}
$$

Lemma 13. If $\nu_{n}(x)=\sum_{k=1}^{n}\left(1-(-1)^{k+m}\right) \alpha_{k} \sin k x$, where all $\alpha_{k}>0$, the sequence $\alpha_{k}$ is monotone, and $m=0,1$. Then

$$
\left|\nu_{n}(x)\right| \leq \frac{c}{|\sin x|}
$$

The proof of this lemma is analogous to the proof of Lemma 12 .

Lemma 14.

$$
\left|\sum_{k=1}^{n} \sin k x\right| \leq \frac{1}{\left|\sin \frac{x}{2}\right|}, \quad \forall n \in \mathbb{N}
$$

Proof. From

$$
\sum_{k=1}^{n} \sin k x=\frac{1}{\sin \frac{x}{2}} \sum_{k=1}^{n} \sin k x \sin \frac{x}{2}
$$

we can easily obtain the equality

$$
\sum_{k=1}^{n} \sin k x=\frac{\sin \frac{n+1}{2} x \sin \frac{n}{2} x}{\sin \frac{x}{2}},
$$

which gives us the desired estimation $\left|\sum_{k=1}^{n} \sin k x\right| \leq \frac{1}{\left|\sin \frac{x}{2}\right|}$.

Lemma 15. The following takes place:

$$
\left|R_{n, N}^{2}\left(f_{2}, x\right)\right| \leq \frac{c(\varepsilon)}{n}, \quad x \in \Delta^{I}(\varepsilon) .
$$


Proof. From (11) and 13) we have

$$
\begin{gathered}
R_{n, N}^{2}\left(f_{2}, x\right)=\frac{2}{\pi} \sum_{\mu=1}^{\infty} \int_{-\pi}^{\pi} \sum_{k=1}^{n} \cos k(x-t) \cos \mu N(u-t) \operatorname{sign} t d t= \\
=\frac{2}{\pi} \sum_{\mu=1}^{\infty} \sin \mu N u \sum_{k=1}^{n} \cos k x \int_{-\pi}^{\pi}(\cos k t \sin \mu N t) \operatorname{sign} t d t+ \\
+\frac{2}{\pi} \sum_{\mu=1}^{\infty} \cos \mu N u \sum_{k=1}^{n} \sin k x \int_{-\pi}^{\pi}(\sin k t \cos \mu N t) \operatorname{sign} t d t= \\
=\frac{4}{\pi} \sum_{\mu=1}^{\infty} \sin \mu N u \sum_{k=1}^{n} \cos k x \int_{0}^{\pi} \cos k t \sin \mu N t d t+ \\
+\frac{4}{\pi} \sum_{\mu=1}^{\infty} \cos \mu N u \sum_{k=1}^{n} \sin k x \int_{0}^{\pi} \sin k t \cos \mu N t d t
\end{gathered}
$$

Using the formula $\sin \alpha \cos \beta=\frac{1}{2}(\sin (\alpha-\beta)+\sin (\alpha+\beta))$ and calculating the integrals from the above equation, we get $\left|R_{n, N}^{2}\left(f_{2}, x\right)\right| \leq$ $\leq\left|R_{n, N}^{2.1}\left(f_{2}, x\right)\right|+\left|R_{n, N}^{2.2}\left(f_{2}, x\right)\right|$, where

$$
\begin{aligned}
& R_{n, N}^{2.1}\left(f_{2}, x\right)=\frac{2}{\pi} \sum_{\mu=1}^{\infty} \sin \mu N u \sum_{k=1}^{n}\left(1-(-1)^{\mu N+k}\right) \cos k x \frac{\mu N}{(\mu N)^{2}-k^{2}}, \\
& R_{n, N}^{2.2}\left(f_{2}, x\right)=\frac{4}{\pi} \sum_{\mu=1}^{\infty} \cos \mu N u \sum_{k=1}^{n}\left(1-(-1)^{\mu N+k}\right) \sin k x \frac{k}{k^{2}-(\mu N)^{2}} .
\end{aligned}
$$

We estimate values $\left|R_{n, N}^{2.1}\left(f_{2}, x\right)\right|$ and $\left|R_{n, N}^{2.2}\left(f_{2}, x\right)\right|$ separately. Begin with $\left|R_{n, N}^{2.1}\left(f_{2}, x\right)\right|$.

$$
\begin{gathered}
R_{n, N}^{2.1}\left(f_{2}, x\right)=\frac{2}{\pi N} \sum_{\mu=1}^{\infty} \frac{\sin \mu N u}{\mu} \sum_{k=1}^{n}\left(1-(-1)^{\mu N+k}\right) \cos k x \frac{1}{1-\frac{k^{2}}{(\mu N)^{2}-k^{2}}}= \\
=\frac{2}{\pi N} \sum_{\mu=1}^{\infty} \frac{\sin \mu N u}{\mu} \sum_{k=1}^{n}\left(1-(-1)^{\mu N+k}\right) \cos k x\left(1+\frac{k^{2}}{(\mu N)^{2}-k^{2}}\right) .
\end{gathered}
$$


We can rewrite it as follows:

$$
\begin{aligned}
R_{n, N}^{2.1}\left(f_{2}, x\right) & =\frac{2}{\pi N} \sum_{\mu=1}^{\infty} \frac{\sin \mu N u}{\mu} \sum_{k=1}^{n} \cos k x+ \\
& +\frac{2}{\pi N} \sum_{\mu=1}^{\infty} \frac{(-1)^{\mu N} \sin \mu N u}{\mu} \sum_{k=1}^{n}(-1)^{k} \cos k x+ \\
& +\frac{2}{\pi N} \sum_{\mu=1}^{\infty} \frac{\sin \mu N u}{\mu} \sum_{k=1}^{n} \cos k x \frac{k^{2}}{(\mu N)^{2}-k^{2}}+ \\
& +\frac{2}{\pi N} \sum_{\mu=1}^{\infty} \frac{(-1)^{\mu N} \sin \mu N u}{\mu} \sum_{k=1}^{n} \cos k x \frac{(-1)^{k} k^{2}}{(\mu N)^{2}-k^{2}}
\end{aligned}
$$

Now we have

$$
\begin{aligned}
\left|R_{n, N}^{2.1}\left(f_{2}, x\right)\right| \leq & \frac{2}{\pi N}\left|\sum_{\mu=1}^{\infty} \frac{\sin \mu N u}{\mu}\right| \cdot\left|\sum_{k=1}^{n} \cos k x\right|+ \\
+ & \frac{2}{\pi N}\left|\sum_{\mu=1}^{\infty} \frac{(-1)^{\mu N} \sin \mu N u}{\mu}\right| \cdot\left|\sum_{k=1}^{n}(-1)^{k} \cos k x\right|+ \\
+ & \left|\frac{2}{\pi N} \sum_{\mu=1}^{\infty} \frac{\sin \mu N u}{\mu} \sum_{k=1}^{n} \cos k x \frac{k^{2}}{(\mu N)^{2}-k^{2}}\right|+ \\
& +\left|\frac{2}{\pi N} \sum_{\mu=1}^{\infty} \frac{(-1)^{\mu N} \sin \mu N u}{\mu} \sum_{k=1}^{n} \cos k x \frac{(-1)^{k} k^{2}}{(\mu N)^{2}-k^{2}}\right|
\end{aligned}
$$

From [4, p. 448] we get

$$
\frac{2}{\pi}\left|\sum_{\mu=1}^{\infty} \frac{\sin \mu N u}{\mu}\right| \leq 1 \text { and } \frac{2}{\pi}\left|\sum_{\mu=1}^{\infty} \frac{(-1)^{\mu N} \sin \mu N u}{\mu}\right| \leq 1 .
$$

Also, from Lemma 7

$$
\left|\sum_{k=1}^{n} \cos k x\right| \leq \frac{1}{|\sin x|}, \quad\left|\sum_{k=1}^{n}(-1)^{k} \cos k x\right| \leq \frac{1}{|\sin x|} .
$$


Using the above estimations we can write

$$
\begin{aligned}
\left|R_{n, N}^{2.1}\left(f_{2}, x\right)\right| & \leq \frac{2}{N|\sin x|}+\frac{2}{\pi N}\left|\sum_{\mu=1}^{\infty} \frac{\sin \mu N u}{\mu} \sum_{k=1}^{n} \cos k x \frac{k^{2}}{(\mu N)^{2}-k^{2}}\right|+ \\
& +\frac{2}{\pi N}\left|\sum_{\mu=1}^{\infty} \frac{(-1)^{\mu N} \sin \mu N u}{\mu} \sum_{k=1}^{n} \cos k x \frac{(-1)^{k} k^{2}}{(\mu N)^{2}-k^{2}}\right| \cdot \quad(24)
\end{aligned}
$$

To estimate the value

$$
\frac{2}{\pi N}\left|\sum_{\mu=1}^{\infty} \frac{\sin \mu N u}{\mu} \sum_{k=1}^{n} \cos k x \frac{k^{2}}{(\mu N)^{2}-k^{2}}\right|
$$

make some transformations:

$$
\begin{gathered}
\frac{2}{\pi N}\left|\sum_{\mu=1}^{\infty} \frac{\sin \mu N u}{\mu} \sum_{k=1}^{n} \cos k x \frac{k^{2}}{(\mu N)^{2}-k^{2}}\right|= \\
=\frac{2}{\pi N}\left|\sum_{\mu=1}^{\infty} \frac{\sin \mu N u}{\mu^{3}} \sum_{k=1}^{n} \cos k x \frac{k^{2}}{N^{2}-\frac{k^{2}}{\mu^{2}}}\right| \leq \\
\leq \frac{2}{\pi N} \sum_{\mu=1}^{\infty} \frac{1}{\mu^{3}}\left|\sum_{k=1}^{n} \cos k x \frac{k^{2}}{N^{2}-\frac{k^{2}}{\mu^{2}}}\right|=\frac{2}{\pi N} \sum_{\mu=1}^{\infty} \frac{1}{\mu^{3}} \mid \frac{n^{2}}{N^{2}-\frac{k^{2}}{\mu^{2}}} \sum_{j=1}^{n} \cos j x- \\
-\sum_{k=1}^{n-1}\left(\frac{(k+1)^{2}}{N^{2}-\frac{(k+1)^{2}}{\mu^{2}}}-\frac{k^{2}}{N^{2}-\frac{k^{2}}{\mu^{2}}}\right) \sum_{j=1}^{k} \cos j x \mid \leq \\
\leq \frac{2}{\pi N} \sum_{\mu=1}^{\infty} \frac{1}{\mu^{3}}\left(\frac{2 n^{2}}{N^{2}-\frac{k^{2}}{\mu^{2}}}\left|\sum_{j=1}^{n} \cos j x\right|+\right. \\
\left.+\sum_{k=1}^{n-1}\left(\frac{(k+1)^{2}}{N^{2}-\frac{(k+1)^{2}}{\mu^{2}}}-\frac{k^{2}}{N^{2}-\frac{k^{2}}{\mu^{2}}}\right)\left|\sum_{j=1}^{k} \cos j x\right|\right) \leq \\
\leq \frac{2}{\pi N|\sin x|} \sum_{\mu=1}^{\infty} \frac{1}{\mu^{3}}\left(\frac{2 n^{2}}{N^{2}-\frac{k^{2}}{\mu^{2}}}-\frac{1}{N^{2}-\frac{1}{\mu^{2}}}\right) \leq
\end{gathered}
$$




$$
\leq \frac{8}{3 \pi N|\sin x|} \sum_{\mu=1}^{\infty} \frac{1}{\mu^{3}} \leq \frac{4}{\pi N|\sin x|} .
$$

Using the similar approach, prove

$$
\frac{2}{\pi N}\left|\sum_{\mu=1}^{\infty} \frac{(-1)^{\mu N} \sin \mu N u}{\mu} \sum_{k=1}^{n} \cos k x \frac{(-1)^{k} k^{2}}{(\mu N)^{2}-k^{2}}\right| \leq \frac{4}{\pi N|\sin x|} .
$$

Now we can rewrite 24 as follows:

$$
\left|R_{n, N}^{2.1}\left(f_{2}, x\right)\right| \leq \frac{1}{N|\sin x|}\left(2+\frac{8}{\pi}\right)=\frac{c}{N|\sin x|}
$$

Now consider $\left|R_{n, N}^{2.2}\left(f_{2}, x\right)\right|$.

$$
\begin{array}{r}
R_{n, N}^{2.2}\left(f_{2}, x\right)=\frac{4}{\pi} \sum_{\mu=1}^{\infty} \cos \mu N u \sum_{k=1}^{n}\left(1-(-1)^{\mu N+k}\right) \sin k x \frac{k}{k^{2}-(\mu N)^{2}}= \\
=\frac{4}{\pi N} \sum_{\mu=1}^{\infty} \frac{\cos \mu N u}{\mu^{2}} \sum_{k=1}^{n}\left(1-(-1)^{\mu N+k}\right) \sin k x \frac{k}{\frac{k^{2}}{\mu^{2} N}-1} . \\
\left|R_{n, N}^{2.2}\left(f_{2}, x\right)\right| \leq \frac{4}{\pi N} \sum_{\mu=1}^{\infty} \frac{1}{\mu^{2}}\left|\sum_{k=1}^{n}\left(1-(-1)^{\mu N+k}\right) \sin k x \frac{k}{\frac{k^{2}}{\mu^{2} N}-1}\right| .
\end{array}
$$

Using the approach we used for estimating $\left|R_{n, N}^{2.1}\left(f_{2}, x\right)\right|$, prove that

$$
\left|R_{n, N}^{2.2}\left(f_{2}, x\right)\right| \leq \frac{c}{N|\sin x|}
$$

From the obtained estimations (25) and (26), and the inequalities $n \leq N / 2$ and $|\sin \varepsilon| \leq|\sin x|$ for $x \in \Delta^{I}(\varepsilon)$, we have 23 .

From 21] and Lemmas 11 and 15 we have

$$
\left|R_{n, N}\left(f_{2}, x\right)\right| \leq \frac{1}{n}(c+c(\varepsilon))=\frac{c(\varepsilon)}{n}, \quad x \in \Delta^{I}(\varepsilon) .
$$

So, Lemma 10 is proved.

6. An estimation for $a_{n}^{(2 n)}\left(f_{2}\right)$. We prove a lemma: 
Lemma 16. The following estimation takes place:

$$
\left|a_{n}^{(2 n)}\left(f_{2}\right)\right| \leq \frac{c}{n}
$$

Proof. Using formula 20 we get (for a $2 \pi$-periodic function $f$ )

$$
a_{n}^{(2 n)}(f)=\frac{1}{2 n} \sum_{k=0}^{2 n-1} f\left(t_{k}\right) \cos \pi k=\frac{1}{2 n} \sum_{k=0}^{2 n-1}(-1)^{k} f(u+\pi k / n) .
$$

As has been mentioned earlier, we can safely assume that $0 \leq u<\pi / n$; so we write

$$
\begin{aligned}
& a_{n}^{(2 n)}\left(f_{2}\right)=1 / 2 n \sum_{k=-n}^{n-1}(-1)^{k} f_{2}(\pi k / n+u), \quad 0 \leq u<\pi / n . \\
& a_{n}^{(2 n)}\left(f_{2}\right)= \frac{1}{2 n}\left(\sum_{k=-n+1}^{-1}(-1)^{k+1}+\sum_{k=1}^{n-1}(-1)^{k}+\right. \\
&\left.+(-1)^{n} f_{2}(u-\pi)+f_{2}(u)\right)=\frac{1}{2 n}\left(f_{2}(u)-(-1)^{n} f_{2}(u)\right) .
\end{aligned}
$$

From this we get

$$
\left|a_{n}^{(2 n)}\left(f_{2}\right)\right| \leq 1 / n
$$

7. Proofs of Theorems 1 and 2, From (4) and (5) we have

$$
\begin{aligned}
& \left|L_{n, N}\left(f_{1}, x\right)-f_{1}(x)\right| \leq\left|S_{n}\left(f_{1}, x\right)-f_{1}(x)\right|+\left|R_{n, N}\left(f_{1}, x\right)\right|, \quad n<N / 2, \\
& \quad\left|L_{n, 2 n}\left(f_{1}, x\right)-f_{1}(x)\right| \leq \\
& \quad \leq\left|S_{n-1}\left(f_{1}, x\right)-f_{1}(x)\right|+\left|R_{n-1,2 n}\left(f_{1}, x\right)\right|+\left|a_{n}^{(2 n)}\left(f_{1}\right)\right|, \quad n=N / 2 .
\end{aligned}
$$

From Lemmas 2, 4, 9 we easily get

$$
\begin{gathered}
\left|L_{n, N}\left(f_{1}, x\right)-f_{1}(x)\right| \leq c / n, \quad x \in[-\pi, \pi], \\
\left|L_{n, N}\left(f_{1}, x\right)-f_{1}(x)\right| \leq c(\varepsilon) / n^{2}, \quad x \in \Delta^{I}(\varepsilon) .
\end{gathered}
$$


Theorem 1 is proved.

Using the same technique, inequalities (4) and (5), and Lemmas 3,10 and 16 , we get

$$
\left|L_{n, N}(f, x)-f(x)\right| \leq c(\varepsilon) / n, \quad x \in \Delta^{I}(\varepsilon) .
$$

So, theorem 2 is also proved.

\section{References}

[1] Bernshtein S. N. On trigonometric interpolation by the method of least squares. Dokl. Akad. Nauk USSR, 1934, vol. 4, pp. 1-5. (in Russian)

[2] Courant R. Differential and Integral Calculus. New Jersey. : WileyInterscience, 1988, vol. 1, 704 p.

[3] Erdös P. Some theorems and remarks on interpolation. Acta Sci. Math. (Szeged), 1950, vol. 12, pp. 11-17.

[4] Fikhtengol'ts G. M. Course of differential and integral calculus. Moscow. : FIZMATLIT, 1969, vol. 1, 656 p. (in Russian)

[5] Kalashnikov M. D. On polynomials of best (quadratic) approximation on a given system of points. Dokl. Akad. Nauk USSR, 1955, vol. 105, pp. 634636. (in Russian)

[6] Krilov V. I. Convergence of algebraic interpolation with respect to the roots of a Chebyshev polynomial for absolutely continuous functions and functions with bounded variation. Dokl. Akad. Nauk USSR, 1956, vol. 107, pp. 362-365. (in Russian)

[7] Magomed-Kasumov M. G. Approximation properties of de la Valle-Poussin means for piecewise smooth functions. Mat. Zametki, 2016, vol. 100, is. 2, pp. 229-244. DOI:10.1134/S000143461607018X

[8] Marcinkiewicz J. Quelques remarques sur l'interpolation. Acta Sci. Math. (Szeged), 1936, vol. 8, pp. 127-130. (in French)

[9] Marcinkiewicz J. Sur la divergence des polynômes d'interpolation. Acta Sci. Math. (Szeged), 1936, vol. 8, pp. 131-135. (in French)

[10] Natanson I. P. On the Convergence of Trigonometrical Interpolation at Equi-Distant Knots. Annals of Mathematics, Second Series, 1944, vol. 45, no. 3, pp. 457-471. DOI:10.2307/1969188.

[11] Nikol'skii S. M. On some methods of approximation by trigonometric sums. Mathematics of the USSR - Izvestiya, 1940, vol. 4, pp. 509-520. (in Russian)

[12] Šarapudinov I. I. On the best approximation and polynomials of the least quadratic deviation. Anal. Math., vol. 9, is. 3. pp. 223-234. 
[13] Zygmund A. Trigonometric Series. Cambridge. : Cambridge University Press, 1959, vol. 1, 747 p.

Received October 11, $201 \%$.

In revised form, December 13, $201 \%$.

Accepted December 15, 2017.

Published online December 27, $201 \%$.

Dagestan scientific center of RAS

45, Gadzhieva st., Makhachkala 367025, Russia

E-mail: hasan.akniyev@gmail.com 